\title{
Antenna Design For Noncoherent Massive MIMO Systems
}

Stephan Bucher, Ahmed N. Ragab, George Yammine, Robert F. H. Fischer, and Christian Waldschmidt resale or redistribution to servers or lists, or reuse of any copyrighted component of this work in other works. 


\title{
Antenna Design For Noncoherent Massive MIMO Systems
}

\author{
Stephan Bucher ${ }^{1}$, Ahmed N. Ragab ${ }^{1}$, George Yammine ${ }^{2}$, Robert F. H. Fischer ${ }^{2}$, Christian Waldschmidt ${ }^{1}$ \\ ${ }^{1}$ Institute of Microwave Engineering, ${ }^{2}$ Institute of Communications Engineering \\ Ulm University, 89081 Ulm, Germany \\ Email: \{stephan.bucher, ahmed.ahmed, george.yammine, robert.fischer, christian.waldschmidt\}@uni-ulm.de
}

\begin{abstract}
Massive MIMO is one of the key technologies that enables an increase in capacity in multi-user MIMO systems. However, these systems suffer from high channel estimation complexity and its degradation due to pilot contamination. An attractive way to overcome the key problems of massive MIMO is to resort to noncoherent detection since no actual channel knowledge is needed at the receiver. In this paper, an appropriate antenna design at the base station is proposed when applying noncoherent detection methods. Thereby, the influence of the main antenna characteristics, namely the radiation pattern, gain, and mutual coupling on the symbol error rate performance is assessed for realistic antennas. The evaluation is based on the COST 2100 channel model, properly adapted to the massive MIMO setting. It is shown that proper antenna design can enhance the symbol error rate performance in the noncoherent case in contrast to coherent detection. More specifically, the base station should be equipped with high gain and highly directional antennas. In addition, mutual coupling between adjacent antenna elements at base station may enhance the system performance when present.
\end{abstract}

\section{INTRODUCTION}

Massive multiple-input/multiple-output (MIMO) systems, where the base station is equipped with a very large number of receive antennas, have attracted more and more attention, e.g., [1]-[3]. However, channel state information at the receiver is required to exploit the benefits of such systems. This involves a high level of effort on channel estimation as the number of channel coefficients to estimate can be very large.

An alternative approach is offered by noncoherent detection, which does not require channel estimation. Inspired from ultrawideband communication systems and applied to the massive MIMO case, noncoherent detection schemes were presented in [4], [5] and assessed for a uniform linear array at the base station, where antennas were employed having omnidirectional and directional characteristics [6]. However, the performance has been evaluated for theoretical (mathematically generated) antenna patterns. Additionally, the evaluation was based on an idealized, geometric channel model, whereas investigations in [7] revealed a significant performance gap for the same setup when using a cluster-based model, in particular the COST 2100 channel model [8], adapted to the massive MIMO setting.

In this paper, the impact of realistic antennas on the performance of noncoherent detection is analyzed covering the main

This work was funded by the Deutsche Forschungsgemeinschaft (DFG, German Research Foundation) - WA 3506/5-1; FI 982/12-1. antenna characteristics, i.e., antenna pattern, gain, and mutual coupling.

This paper is organized as follows. In Sec. II the system model including a brief review of the COST 2100 channel model and the noncoherent detection scheme is introduced. Additionally, a proper model to account for mutual coupling of the antennas at the base station side is presented. The numerical results are shown and discussed in Sec. III. Conclusions are drawn in Sec. IV.

\section{Noncoherent MASsive MiMO System Model}

Throughout this paper, a multi-user uplink scenario is considered (see Fig. 1), where $N_{\mathrm{u}}$ users simultaneously transmit to a central base station (BS) equipped with a very large number of receive antennas $N_{\mathrm{rx}} \gg N_{\mathrm{u}}$. At the user side, single omnidirectional antennas are employed, whereas the antennas at the BS can have a directional pattern. The data symbols $a_{k, u}$ at each time step $k$ and for each user $u$ are drawn from an $M$-ary PSK constellation $\mathcal{M}=\left\{\mathrm{e}^{\mathrm{j} 2 \pi \cdot i / M} \mid i=0,1, \ldots, M-1\right\}$ and are differentially encoded (resulting in the transmit symbols $b_{k, u}$ ). At the base station, noncoherent detection methods are applied.

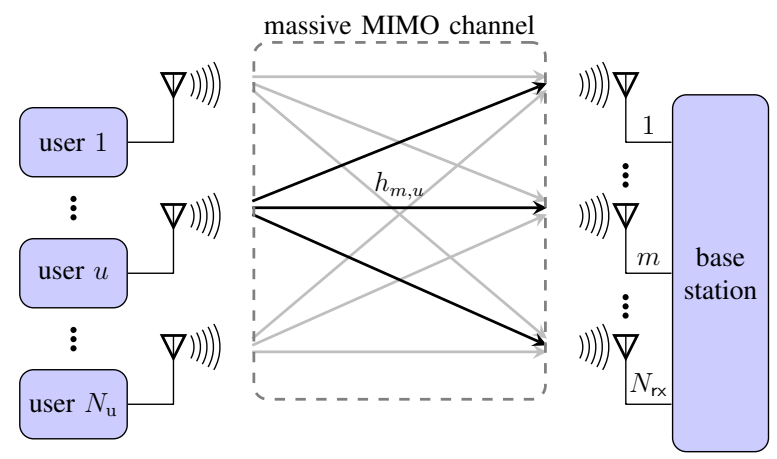

Fig. 1. Illustration of the multi-user massive MIMO uplink system.

\section{A. Massive MIMO Channel Model}

The massive MIMO channel is modeled by the well-known COST 2100 channel model [8], [9]. This geometry-based stochastic channel model emulates physical scattering objects by placing clusters randomly in the topological simulation environment. Each cluster consists of groups of multipath 


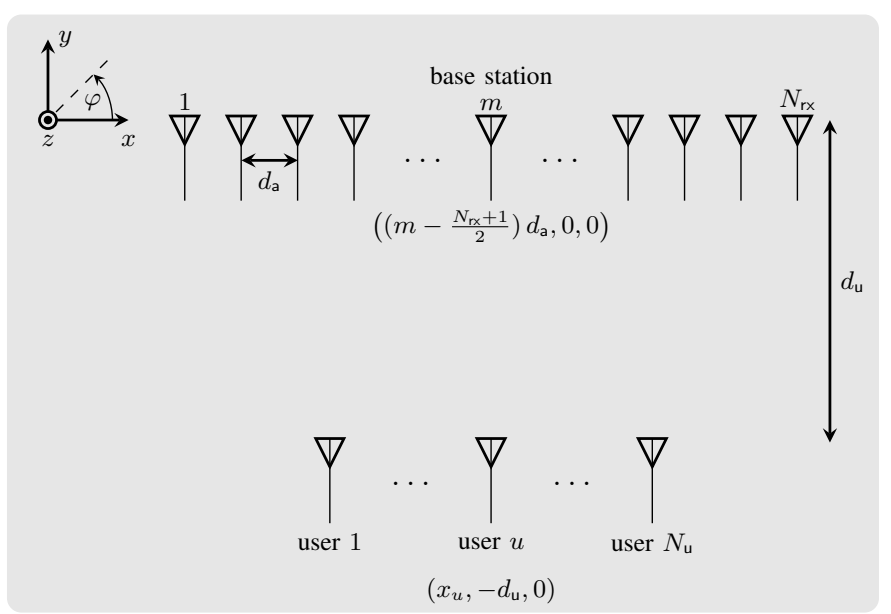

Fig. 2. User and base station arrangement using the extended COST 2100 channel model. The shaded area $(\rightarrow$ ) denotes the topological simulation region.

components and its parameters are calculated from the geometry of the simulation area. In general, there exist four kinds of clusters. A distinction is made according to local clusters (scatterers around the users and the BS), single/twin clusters (scattering mechanism with one or multiple objects), and common clusters (users/BS antennas share the same set of clusters). Besides the multipath propagation, a line-of-sight (LOS) path between users and BS can be considered as well. The activity of each cluster (except local clusters) is controlled by visibility regions (VRs), which the clusters are assigned to. When the user is located in a specific VR, the corresponding clusters are active. As the initial COST 2100 channel model supports conventional MIMO systems with small and compact arrays, the extended version is taken from [7] to investigate the present massive MIMO system comprising of a physical large array. The arrangement of the users and the BS using the extended channel model is depicted in Fig. 2. At the BS a uniform linear array consisting of $N_{\text {rx }}$ antennas with an inter-element distance $d_{\mathrm{a}}$ is assumed. The users are placed at distance $d_{\mathrm{u}}$ to the BS and are positioned along a straight line in the $x$-direction. The users as well as the BS are assumed to be located in the azimuthal plane.

Given the antenna pattern $C_{\mathrm{rx}}(\varphi, \theta)$ and the antenna gain $G_{\mathrm{rx}}$ at the BS side, the channel coefficient between user $u$ and BS antenna $m$ can be expressed by [7]

$$
\begin{aligned}
h_{m, u}= & a_{m, u}^{\mathrm{LOS}} C_{\mathrm{rx}}\left(\varphi_{m, u}^{\mathrm{LOS}}, \theta_{m, u}^{\mathrm{LOS}}\right) \sqrt{G_{\mathrm{rx}}} \\
& +\sum_{i} a_{i, m, u}^{\mathrm{MPC}} C_{\mathrm{rx}}\left(\varphi_{i, m, u}^{\mathrm{MPC}}, \theta_{i, m, u}^{\mathrm{MPC}}\right) \sqrt{G_{\mathrm{rx}}},
\end{aligned}
$$

where $a_{m, u}^{\mathrm{LOS}}$ is the complex amplitude of the LOS component with the corresponding direction of arrivals (DOA) $\varphi_{m, u}^{\mathrm{LOS}}$ and $\theta_{m, u}^{\mathrm{LOS}}$. The complex amplitude of the $i$-th multipath component originating from the currently active clusters is denoted by $a_{i, m, u}^{\mathrm{MPC}}$ and its DOAs by $\varphi_{i, m, u}^{\mathrm{MPC}}$ and $\theta_{i, m, u}^{\mathrm{MPC}}$, respectively. Both $a_{m, u}^{\mathrm{LOS}}$ and $a_{i, m, u}^{\mathrm{MPC}}$ depend on the length of the propagation path. However, since clusters are randomly distributed in the simulation area, $a_{i, m, u}^{\mathrm{MPC}}$ becomes random for different channel realizations, whereas $a_{m, u}^{\mathrm{LOS}}$ is deterministic, since the length of the propagation path remains unchanged.

The power distribution of each individual user over the receive antennas (power-space profile, PSP) is characterized by the squared magnitude of the channel coefficients averaged over different channel realizations

$$
P_{m, u} \stackrel{\text { def }}{=} \mathrm{E}\left\{\left|h_{m, u}\right|^{2}\right\},
$$

i.e., $P_{m, u}$ is the average received power at the $m$-th receive antenna induced by user $u$.

\section{B. Noncoherent Detection}

At the receiver, decision-feedback differential detection in combination with noncoherent decision-feedback equalization (DFDD/nDFE) is applied [5]. For this purpose, the receive signal over a burst of $N_{\mathrm{bl}}$ symbols is considered. Assuming that the channel coefficients are constant over an entire burst, the $N_{\mathrm{rx}} \times N_{\mathrm{bl}}$ receiving block $\boldsymbol{R}$ is given by

$$
\boldsymbol{R}=\boldsymbol{H} \boldsymbol{B}+\boldsymbol{N},
$$

where $\boldsymbol{H}$ indicates the $N_{\mathrm{rx}} \times N_{\mathrm{u}}$ channel matrix containing the complex-valued channel coefficients $h_{m, u}$. The matrix $\boldsymbol{B}$ of size $N_{\mathrm{u}} \times N_{\mathrm{bl}}$ consists of the transmit symbols $b_{k, u}$ of each user $u$ over the block and the matrix $N$ of size $N_{\mathrm{rx}} \times N_{\mathrm{bl}}$ gathers the circular-symmetric zero-mean complex Gaussian noise $n_{m, k}$ with variance $\sigma_{\mathrm{n}}^{2}$

The noncoherent detection scheme is based on the $N_{\mathrm{bl}} \times N_{\mathrm{bl}}$ correlation matrix

$$
\boldsymbol{Z}_{u} \stackrel{\text { def }}{=} \boldsymbol{R}^{\mathrm{H}} \boldsymbol{W}_{u} \boldsymbol{R}
$$

where $\boldsymbol{W}_{u} \stackrel{\text { def }}{=} \operatorname{diag}\left(w_{1, u}, \ldots, w_{1, N_{\text {rx }}}\right)$ is the user-specific diagonal weighting matrix, which is numerically optimized for maximum signal-to-interference-plus-noise ratio (SINR) [6]. The SINR purely depends on the power-space profiles $P_{m, u}$ and the noise variance $\sigma_{\mathrm{n}}^{2}$.

\section{Mutual Coupling Model}

Closely spaced antennas at the BS can disturb the radiation pattern and the input impedance of each array element as the electric field generated from one antenna changes the current distribution of the other antennas [10], [11]. In order to take this mutual coupling effect into account, the channel matrix is multiplied by an $N_{\mathrm{rx}} \times N_{\mathrm{rx}}$ coupling matrix $\boldsymbol{C}_{\text {coupl }}$ at the receiver side such as

$$
\boldsymbol{H}_{\text {coupl }}=\boldsymbol{C}_{\text {coupl }} \boldsymbol{H} \text {. }
$$

Using fundamental electromagnetics and circuit theory, the coupling matrix can be written as in [11]-[13]

$$
\boldsymbol{C}_{\text {coupl }}=\left(Z_{\mathrm{A}}+Z_{\mathrm{L}}\right)\left(\boldsymbol{Z}+Z_{\mathrm{L}} \boldsymbol{I}\right)^{-1},
$$

where $Z_{\mathrm{A}}$ is the antenna impedance in isolation (no coupling present), $Z_{\mathrm{L}}$ is the load impedance and $Z$ denotes the $N_{\mathrm{rx}} \times N_{\mathrm{rx}}$ mutual impedance matrix. The matrix $\boldsymbol{I}$ indicates the $N_{\mathrm{rx}} \times N_{\mathrm{rx}}$ identity matrix. In order to provide an impedance match and guarantee maximum power transfer, the load impedance $Z_{\mathrm{L}}$ is selected as the complex conjugate of the antenna impedance $Z_{\mathrm{A}}$ in isolation. 


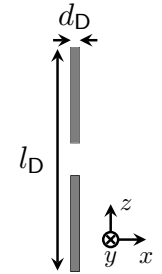

(a) $\frac{\lambda}{2}$-dipole.

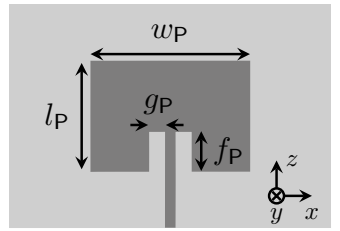

(b) Inset-fed patch antenna.

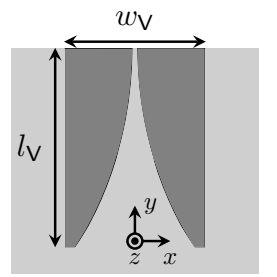

(c) Vivaldi antenna.
Fig. 3. Utilized antenna types (and their alignment) at the BS to assess the performance of the noncoherent detection scheme. Metallization depicted in and substrate in

TABLE I

DIMENSIONS AND CHARACTERISTICS OF UTILIZED ANTENNAS AT THE BS

Antenna dimensions

\begin{tabular}{|l||c|c|c|c|}
\hline \multirow{2}{*}{ dipole } & $l_{\mathrm{D}}$ & $d_{\mathrm{D}}$ & & \\
\cline { 2 - 5 } & $150 \mathrm{~mm}$ & $5 \mathrm{~mm}$ & & \\
\hline \hline \multirow{2}{*}{ patch } & $l_{\mathrm{P}}$ & $w_{\mathrm{P}}$ & $g_{\mathrm{P}}$ & $f_{\mathrm{P}}$ \\
\hline & $65.5 \mathrm{~mm}$ & $92.1 \mathrm{~mm}$ & $2 \mathrm{~mm}$ & $22.9 \mathrm{~mm}$ \\
\hline \hline \multirow{2}{*}{ Vivaldi } & $l_{\mathrm{V}}$ & $w_{\mathrm{V}}$ & & \\
\cline { 2 - 5 } & $314.5 \mathrm{~mm}$ & $208 \mathrm{~mm}$ & & \\
\hline
\end{tabular}

\begin{tabular}{|l||c|c|c|}
\hline \multicolumn{1}{|c||}{ antenna characteristics } & dipole & patch & Vivaldi \\
\hline \hline operating frequency $f_{\mathrm{c}}$ & \multicolumn{3}{|c|}{$1 \mathrm{GHz}$} \\
\hline radiation pattern $C_{\mathrm{rx}}(\varphi, \theta)$ & \multicolumn{3}{|c|}{ see Fig. 4} \\
\hline azimuth $3 \mathrm{~dB}$ beamwidth $\varphi_{3 \mathrm{~dB}}$ & - & $77^{\circ}$ & $51^{\circ}$ \\
\hline antenna gain $G_{\mathrm{rx}}$ & $2.15 \mathrm{dBi}$ & $5 \mathrm{dBi}$ & $8.4 \mathrm{dBi}$ \\
\hline antenna impedance $Z_{\mathrm{A}}$ & $73+\mathrm{j} 42.5 \Omega$ & $50 \Omega$ & $91.2+\mathrm{j} 6.9 \Omega$ \\
\hline
\end{tabular}

\section{Simulation Results}

The performance of the noncoherent detection scheme is assessed for three different antenna types at the BS, namely for $\lambda / 2$-dipole, inset-fed patch, and Vivaldi antennas as depicted in Fig. 3. These antennas are essentially different regarding their antenna pattern, gain, and mutual coupling, thus providing a solid basis to investigate their impact on the noncoherent detection. The antennas shown in Fig. 3 are designed for an operating frequency of $1 \mathrm{GHz}$ following the design guide in [10], [14], [15]. Their dimensions and characteristics are summarized in Table I. In case of the patch and the Vivaldi antenna an FR-4 substrate of height $h=1.6 \mathrm{~mm}$ and of permittivity $\varepsilon_{\mathrm{r}}=4.3$ is used.

Simulations are carried out for $N_{\mathrm{u}}=3$ users and a base station consisting of $N_{\mathrm{rx}}=100$ antennas arranged as a uniform linear array. As scenario, an indoor hall environment is assumed. In this case the extended COST 2100 channel model is parameterized properly according to [9]. It was shown in [7] that the set of clusters has almost no impact on the overall performance. Therefore, simulations are conducted for local clusters only and LOS propagation is omitted. All users and the BS are arranged as illustrated in Fig. 2. At the BS, the inter-element distance amounts to one wavelength $\left(d_{\mathrm{a}}=\lambda\right)$, while the operating frequency is set to $1 \mathrm{GHz}$. Users are placed at a distance of $d_{\mathrm{u}}=5 \mathrm{~m}$ to the BS. User 1 and user 3 are

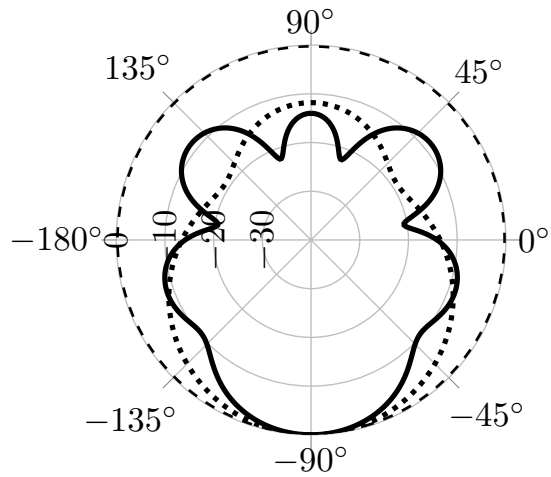

$$
\text { - - - Dipole..... Patch — Vivaldi }
$$

Fig. 4. Azimuth radiation pattern $C_{\mathrm{rx}}\left(\varphi, \theta=90^{\circ}\right)$ (in dB) of investigated antennas utilized at the base station side. The main lobe $\left(\right.$ at $\left.\varphi=-90^{\circ}\right)$ is directed towards the users.

TABLE II

PARAMETERIZATION OF COST 2100 CHANNEL MODEL AND NONCOHERENT DETECTION FOR REFERENCE SCENARIO

COST 2100 channel model

\begin{tabular}{|c|c|}
\hline number of users $N_{\mathrm{u}}$ & 3 \\
\hline user distance $d_{\mathrm{u}}$ & $5 \mathrm{~m}$ \\
\hline number of BS antennas $N_{\text {rx }}$ & 100 \\
\hline operating frequency $f_{\mathrm{c}}$ & $1 \mathrm{GHz}$ \\
\hline BS antenna spacing $d_{\mathrm{a}}$ & $\lambda(=0.3 \mathrm{~m})$ \\
\hline BS antenna type & see Fig. 3 \\
\hline cluster types & local \\
\hline coordinates of users $\left(x_{u}, y_{u}, z_{u}\right)$ & $\begin{array}{c}\left(-30.5 \lambda,-d_{\mathrm{u}}, 0\right) \\
\left(x,-d_{\mathrm{u}}, 0\right) \\
\left(30.5 \lambda,-d_{\mathrm{u}}, 0\right)\end{array}$ \\
\hline $\begin{array}{l}\text { coordinates of } m \text {-th } \\
\text { BS antenna }\left(x_{m}, y_{m}, z_{m}\right)\end{array}$ & $\left(\left(m-\frac{N_{\mathrm{rx}}+1}{2}\right) d_{\mathrm{a}}, 0,0\right)$ \\
\hline $\begin{array}{l}\text { number of different channel } \\
\text { realizations } N_{\text {ch }} \text { per } x \text {-position } \\
\text { of user } 2\end{array}$ & $300,000^{1}$ \\
\hline $\begin{array}{l}\text { channel normalization } \\
\text { (power control) }\end{array}$ & $\left\|\boldsymbol{h}_{u}\right\|_{2}^{2}=1$ \\
\hline \multicolumn{2}{|c|}{ Noncoherent detection } \\
\hline modulation alphabet & 4-ary DPSK \\
\hline block length $N_{\mathrm{bl}}$ & 200 \\
\hline SNR $E_{\mathrm{s}} / N_{0}$ & $14 \mathrm{~dB}$ \\
\hline power control & $\sum_{m=1}^{N_{r x}} P_{m, u}=1$ \\
\hline
\end{tabular}

positioned in such a way that they face the 20th and the 80th BS antenna, respectively, whereas the $x$-position of user 2 is varied.

The performance is measured by the symbol error rate (SER) as a function of the $x$-position of user 2 within a specified range. For each position of user $2, N_{\mathrm{ch}}=300,000^{1}$

\footnotetext{
${ }^{1}$ In order to reduce the complexity, the following was implemented. First, 10,000 different channel realizations of the channel matrix $\tilde{\boldsymbol{H}}$ are generated using the COST 2100 model. Next, the covariance matrices $\boldsymbol{Q}_{u}=\mathrm{E}\left\{\tilde{\boldsymbol{h}}_{u} \tilde{\boldsymbol{h}}_{u}^{\mathrm{H}}\right\}$ are constructed for each user $u$. Finally, 300,000 different channel coefficient vectors of $\boldsymbol{h}_{u}$ are drawn as circularly-symmetric zero-mean complex Gaussian random vectors according to $\boldsymbol{h}_{u} \sim \mathcal{C N}\left(\mathbf{0}, \boldsymbol{Q}_{u}\right)$.
} 


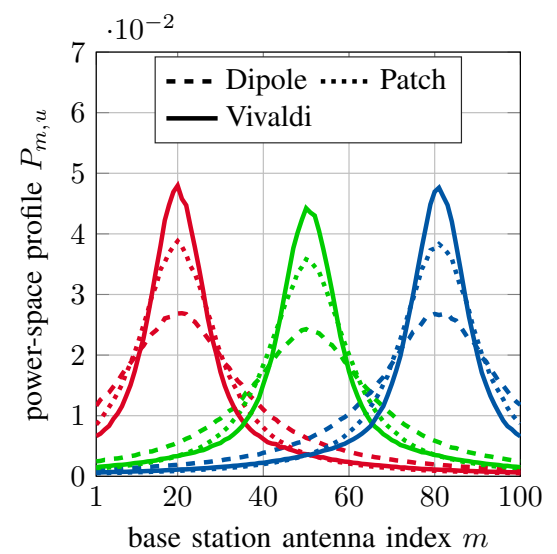

(a) Power-space profile $P_{m, u}$ at zero $x$-position of user 2 .

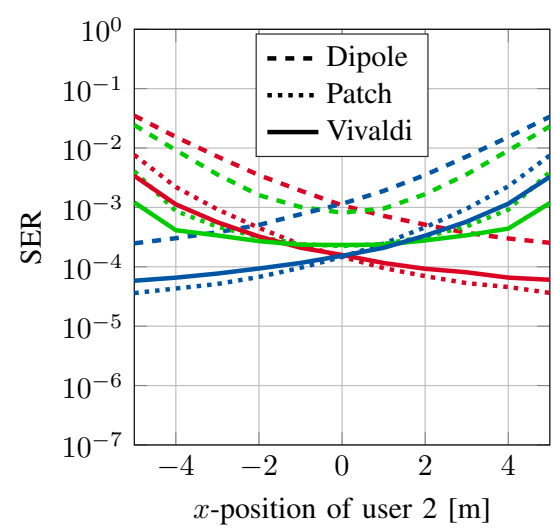

(b) Symbol error rate vs. $x$-position of user 2 using DFDD/nDFE.

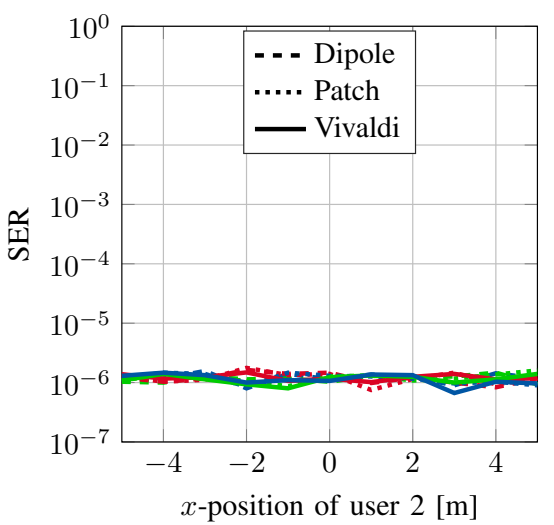

(c) Symbol error rate vs. $x$-position of user 2 using BLAST with perfect channel knowledge at the receiver.

Fig. 5. Performance comparison employing different antenna patterns $C_{\mathrm{rx}}(\varphi, \theta)$ at the base station. Colors correspond to users: user 1 ( - ), user $2(-\longrightarrow)$, user 3 ( $)$. Parameterization according to Table II.

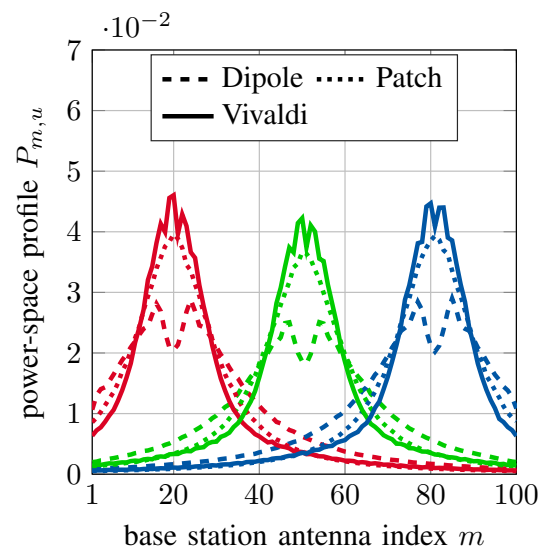

(a) Power-space profile $P_{m, u}$ at zero $x$-position of user 2 .

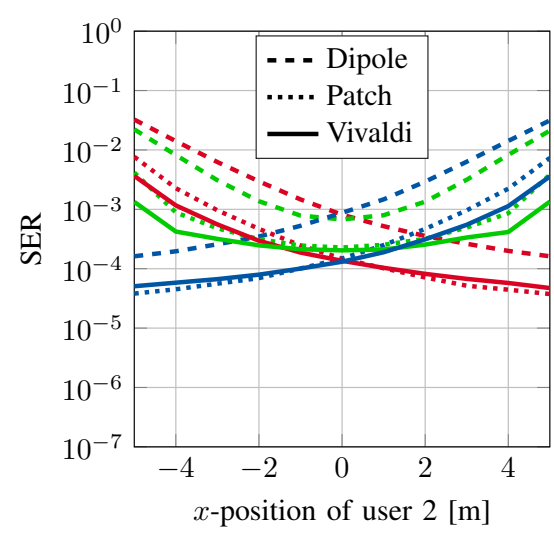

(b) Symbol error rate vs. $x$-position of user 2 using DFDD/nDFE.

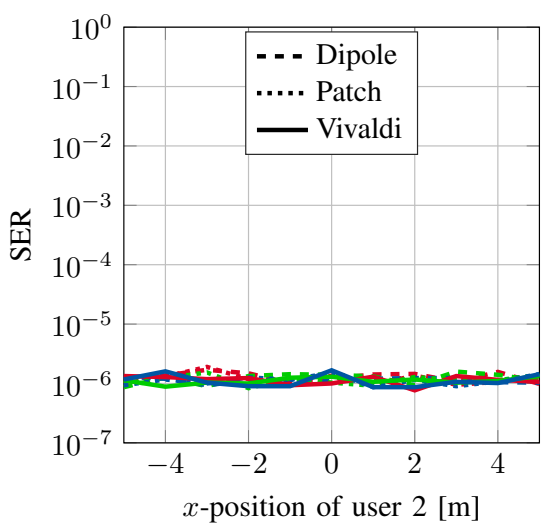

(c) Symbol error rate vs. $x$-position of user 2 using BLAST with perfect channel knowledge at the receiver.

Fig. 6. Performance comparison employing different antenna patterns $C_{\mathrm{rx}}(\varphi, \theta)$ at the base station and including the coupling effect by means of (5). Colors correspond to users: user $1(-)$, user $2(-)$, user 3 ( $(-)$. Parameterization according to Table II.

different channel matrices are acquired for the given setup. It is assumed that the transmit power of each user is adjusted in such a way that each user induces the same sum power at the receiving antenna array. This power control is equivalently modeled as a columnwise normalization of the channel matrix $\boldsymbol{H}$ according to $\left\|\boldsymbol{h}_{u}\right\|_{2}^{2}=1$. This immediately yields $\sum_{m=1}^{N_{\text {rx }}} P_{m, u}=1$ for each user $u$ since $\sum_{m=1}^{N_{\text {rx }}} P_{m, u}=$ $\sum_{m=1}^{N_{\text {rx }}} \mathrm{E}\left\{\left|h_{m, u}\right|^{2}\right\}=\mathrm{E}\left\{\sum_{m=1}^{N_{\text {rx }}}\left|h_{m, u}\right|^{2}\right\}=\mathrm{E}\left\{\left\|\boldsymbol{h}_{u}\right\|_{2}^{2}\right\}=1$. Each user transmits differentially encoded 4PSK symbols. The block length amounts to $N_{\mathrm{bl}}=200$. The signal-to-noise ratio (SNR) is defined as the ratio of the transmitted energy per PSK symbol $E_{\mathrm{s}}$ and the noise power spectral density $N_{0}$. Unless stated otherwise $E_{\mathrm{s}} / N_{0} \widehat{=} 14 \mathrm{~dB}$. The PSPs required for the noncoherent detection via DFDD/nDFE are obtained from the channel coefficients as in (2). A summary of the noncoherent detection and the relevant parameterization of the COST 2100 channel model is given in Table II.

\section{A. Antenna Pattern}

The SER averaged over $N_{\mathrm{ch}}=300,000$ channel realizations for the three different antenna types and for each user are shown in Fig. 5b considering solely the antenna pattern (see respective radiation pattern $C_{\mathrm{rx}}(\varphi, \theta)$ in the azimuthal plane in Fig. 4). It can be noticed, that the SER is dependent on the position of user 2 regardless of what antenna type is utilized at the BS. Best overall performance is achieved by the patch antenna, whereas the Vivaldi antenna has the lowest dependency of the positioning of user 2 . The present results can be explained from the PSPs, which are depicted in Fig. 5a, when the user 2 is located in the middle of the remaining 
users. The smaller the $3 \mathrm{~dB}$ beamwidth of the antenna type, the more receive power is focused on a smaller part of the BS. This behavior reduces the overlapping of the individual PSP of each user and thus enhances the user separability for the noncoherent detection scheme. However, the stronger the power-focusing effect, the less the degrees of freedom for the noncoherent detection. This is the reason why a slight performance loss can be observed in case of the Vivaldi antenna despite its smallest $3 \mathrm{~dB}$ beamwidth of $51^{\circ}$.

For comparison, Fig. 5c shows the results when applying BLAST [16] with perfect channel knowledge at the receiver. It can be observed that the performance of BLAST is independent of both antenna type and user 2 position. Furthermore, there is a performance gap compared to noncoherent detection. However, the channel estimation error for BLAST has not been taken into account, and the given results illustrate rather a performance limit.

\section{B. Antenna Coupling}

Besides the radiation pattern, the impact of coupling of adjacent antenna elements is of major interest. For this purpose, the channel matrix is multiplied by a coupling matrix as in (5). Assuming a transmit power control is applied as above, the columns of the resulting channel matrix are normalized to $\left\|\boldsymbol{h}_{\text {coupl }, u}\right\|_{2}^{2}=1$. The impedance matrix $\boldsymbol{Z}$ in (6) is calculated from analytic expressions for the dipole antenna [10]. However, for the patch and the Vivaldi antenna such equations do not exist. Therefore, assuming coupling between a small number of neighboring antennas only, $5 \times 5$ impedance matrices $Z_{\mathrm{bl}}$ of the prior mentioned antennas are obtained from EM simulations considering five antennas along a straight line distanced by $d_{\mathrm{a}}=\lambda$ (see Fig. 7). The impedance matrix $Z$ is then reconstructed as diagonal block matrix from $\boldsymbol{Z}_{\mathrm{bl}}$ as $\boldsymbol{Z}=\operatorname{diag}\left(\boldsymbol{Z}_{\mathrm{bl}, 1}, \ldots, \boldsymbol{Z}_{\mathrm{bl}, N_{\mathrm{rx}} / 5}\right)$.

Fig. 6b shows the SER when coupling is applied at the BS side. In comparison to the results in Fig. 5b, where no mutual coupling is present, minimal enhancement can be observed in particular for the dipole and Vivaldi antenna. Regarding the PSPs (see Fig. 6a), disturbances are noticeable, especially around the receive antennas the users are closest to. The coupling disturbs the antenna pattern in such a way that notches are generated in the direction to the users. These notches are then reflected in the PSPs and appear mainly for the dipole and Vivaldi antenna. Furthermore, the PSPs are widened at the BS antennas closest to each respective user. This in turn improves the performance of noncoherent detection. No remarkable changes can be observed when using BLAST as shown in Fig. 6c.

Consequently, mutual coupling can be desirable for the noncoherent detection scheme. However, only a moderate antenna spacing of $\lambda$ has been investigated, and for smaller element distances the coupling effect can be even higher. But reducing the element distance causes broadening of the PSP and in turn, degrades performance as can be seen in [7].

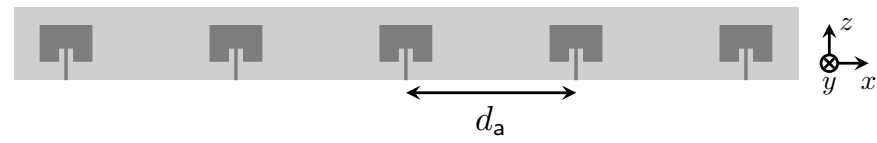

(a) Patch antenna.

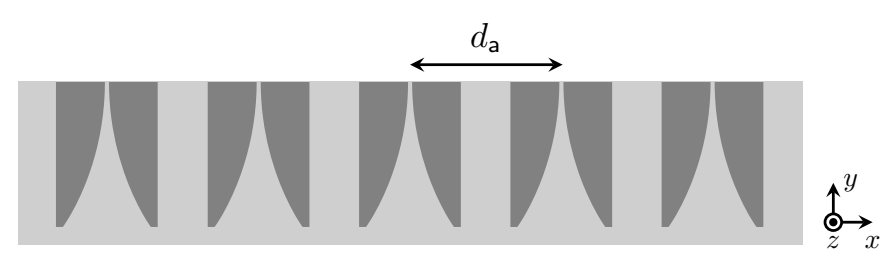

(b) Vivaldi antenna.

Fig. 7. Antenna arrangement in order to obtain impedance matrices for (6). Metallization is depicted in and substrate in $\square$.

\section{Antenna Gain}

In Sec. III-A, the performance of the antenna types has been evaluated purely on the antenna pattern as the channel has been normalized in such a way that the total received power remains the same regardless of the utilized antenna type and its gain. In order to cover the gain effect, the channel normalization (transmit power control) is adapted to $\left\|\boldsymbol{h}_{u}\right\|_{2}^{2}=G_{\mathrm{AUT}, \mathrm{rx}} / G_{\mathrm{D}, \mathrm{rx}}$, where $G_{\mathrm{AUT}, \mathrm{rx}}$ is the gain of the antenna under test and $G_{\mathrm{D}, \mathrm{rx}}$ denotes the gain of the dipole antenna acting as reference. The gain values of the individual antennas can be found in Table I. The results are depicted in Fig. 8, where in this case the Vivaldi antenna reveals best overall performance among the investigated antenna types (note that SNR is reduced to $E_{\mathrm{s}} / N_{0} \widehat{=} 10 \mathrm{~dB}$ ). This behavior originates from the high induced receive power due to the high antenna gain. However, it is remarkable that only in areas where low interferences occur, i.e., the users are sufficiently spatially separated and the overlapping of the PSPs is less, substantial gains are achievable.

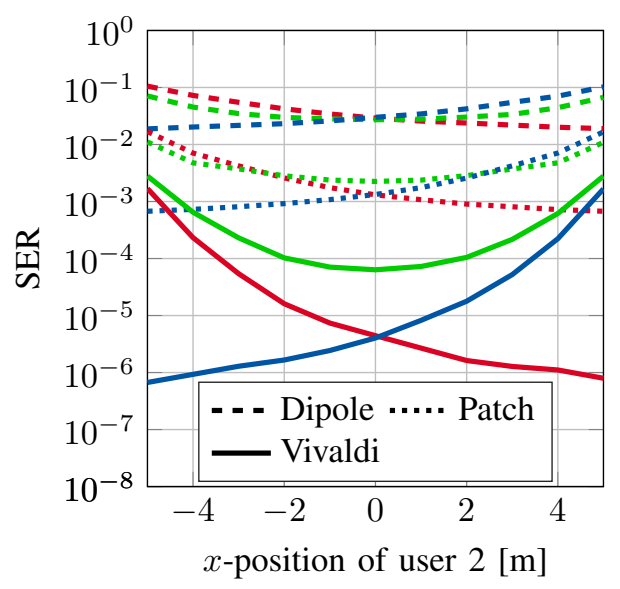

Fig. 8. Symbol error rate vs. $x$-position of user 2 using DFDD/nDFE and employing different antenna patterns $C_{\mathrm{rx}}(\varphi, \theta)$ at the base station. Colors correspond to users: user $1(\square)$, user $2(\stackrel{\square}{\square}$, user 3 ( $(\longrightarrow)$. Parameterization according to Table II, but normalization of channel and PSP adapted to $\left\|\boldsymbol{h}_{u}\right\|_{2}^{2}=G_{\mathrm{AUT}, \mathrm{rx}} / G_{\mathrm{D}, \mathrm{rx}}$ and $\sum_{m=1}^{N_{\mathrm{rx}}} P_{m, u}=G_{\mathrm{AUT}, \mathrm{rx}} / G_{\mathrm{D}, \mathrm{rx}}$, respectively, in order to cover the gain effect. The SNR amounts to $E_{\mathrm{s}} / N_{0} \widehat{=} 10 \mathrm{~dB}$ 


\section{CONCLUSION}

In this paper, an appropriate antenna design is proposed at the base station in case of a noncoherent massive MIMO system. To this end, the impact on the symbol error rate performance of three antenna types has been analyzed when applying noncoherent detection, whereby each of the utilized antennas have significantly different characteristics according to their antenna pattern, gain, and mutual coupling. At the base station, a uniform linear array has been assumed and the investigations are based on a cluster-based channel model, in particular the COST 2100 channel model. It has been shown that the antenna pattern mainly affects the user separability as the receive power is focused on a smaller part of the antenna array at the BS. Mutual coupling between adjacent elements disturbs the PSPs, however, it can have a positive impact on the performance. A substantial increase in performance is obtained when the antenna gain is high and the users are separated sufficiently. Overall, antennas at the base station side should exhibit a highly directional antenna pattern with high gain when applying noncoherent detection. If mutual coupling is additionally present, better performance may be achieved.

\section{REFERENCES}

[1] F. Rusek, D. Persson, B. K. Lau, E. G. Larsson, T. L. Marzetta O. Edfors, and F. Tufvesson, "Scaling Up MIMO: Opportunities and Challenges with Very Large Arrays," IEEE Signal Processing Magazine, vol. 30, no. 1, pp. 40-60, Jan. 2013.

[2] E. G. Larsson, O. Edfors, F. Tufvesson, and T. L. Marzetta, "Massive MIMO for Next Generation Wireless Systems," IEEE Communications Magazine, vol. 52, no. 2, pp. 186-195, Feb. 2014.

[3] S. Yang and L. Hanzo, "Fifty Years of MIMO Detection: The Road to Large-Scale MIMOs," IEEE Communications Surveys \& Tutorials, vol. 17, no. 4, pp. 1941-1988, 2015

[4] A. Schenk and R. F. H. Fischer, "Noncoherent Detection in Massive MIMO Systems," in 17th International ITG Workshop on Smart Antennas (WSA), Mar. 2013, pp. 1-8.
[5] R. F. H. Fischer and M. Bense, "Noncoherent Decision-Feedback Equalization in Massive MIMO Systems," in International Zurich Seminar on Communications (IZS), Feb. 2014, pp. 112-115.

[6] G. Yammine, R. F. H. Fischer, and C. Waldschmidt, "On the Influence of the Antenna Pattern in Noncoherent Massive MIMO Systems," in International Symposium on Wireless Communication Systems (ISWCS), Aug. 2015, pp. 391-395.

[7] S. Bucher, G. Yammine, R. F. H. Fischer, and C. Waldschmidt, "Influence of Channel Parameters on Noncoherent Massive MIMO Systems," in 22nd International ITG Workshop on Smart Antennas (WSA), Mar. 2018.

[8] L. Liu, C. Oestges, J. Poutanen, K. Haneda, P. Vainikainen, F. Quitin, F. Tufvesson, and P. D. Doncker, "The COST 2100 MIMO Channel Model," IEEE Wireless Communications, vol. 19, no. 6, pp. 92-99, Dec. 2012.

[9] R. Verdone and A. Zanella, Eds., Pervasive Mobile and Ambient Wireless Communications. Springer London, 2012.

[10] C. A. Balanis, Antenna Theory: Analysis and Design, 2nd ed. Wiley, 1997.

[11] B. Clerckx, C. Craeye, D. Vanhoenacker-Janvier, and C. Oestges, "Impact of Antenna Coupling on $2 \times 2$ MIMO Communications," IEEE Transactions on Vehicular Technology, vol. 56, no. 3, pp. 1009-1018, May 2007.

[12] T. Svantesson and A. Ranheim, "Mutual Coupling Effects on the Capacity of Multielement Antenna Systems," in International Conference on Acoustics, Speech, and Signal Processing (ICASSP), May 2001, pp. 2485-2488.

[13] S. Durrani and M. E. Bialkowski, "Effect of Mutual Coupling on the Interference Rejection Capabilities of Linear and Circular Arrays in CDMA Systems," IEEE Transactions on Antennas and Propagation, vol. 52, no. 4, pp. 1130-1134, Apr. 2004

[14] J. Volakis, Antenna Engineering Handbook, Fourth Edition, 4th ed. McGraw-Hill, 2007.

[15] J. Shin and D. Schaubert, "A Parameter Study of Stripline-Fed Vivald Notch-Antenna Arrays," IEEE Transactions on Antennas and Propagation, vol. 47, no. 5, pp. 879-886, May 1999.

[16] G. J. Foschini, D. Chizhik, M. J. Gans, C. Papadias, and R. A. Valenzuela, "Analysis and Performance of Some Basic Space-Time Architectures," IEEE Journal on Selected Areas in Communications, vol. 21, no. 3, pp. 303-320, Apr. 2003. 